\title{
Hib epiglottitis despite fully vaccinated status
}

\author{
Daniel Chandler, Martin Connor and David Breen
}

\begin{abstract}
The introduction of an effective vaccine has markedly reduced the incidence of invasive Haemophilus influenzae type b (Hib) disease. However, vaccination failure can occur, and this report describes one such case in a previously healthy 4 -year-old girl, who became severely unwell with Hib epiglottitis. She had received a full course of Hib vaccine administered via the buttocks. This site has been associated with reduced immunogenicity compared to vaccination by injection into the thigh or deltoid muscles. Current recommendations are to avoid gluteal injection for all vaccinations.
\end{abstract}

Keywords

epiglottitis; Haemophilus influenzae type b; vaccination technique.
D Chandler, $M B C h B, M P H$, specialty registrar in public health; D Breen, MSc, FFPH, DCH, DObstRCPI, consultant in public health medicine, Directorate of Public Health \& Strategic Planning; M Connor, MSc, FRCPath, FFPH, $D T M \& H$, consultant microbiologist, Department of Microbiology, NHS Dumfries \& Galloway, Dumfries.

Address for correspondence

Daniel Chandler, NHS Dumfries \& Galloway, Directorate of Public Health \& Strategic Planning, Crichton Hall, Bankend Road, Dumfries, DG1 4TG. E-mail: d.chandler@nhs.net

Submitted: 25 November 2008; final acceptance: 13 February 2009.

(OBritish Journal of General Practice 2009; 59: 597-598. DOI: 10.3399/bjgp09X453828

\section{INTRODUCTION}

Haemophilus influenzae type b (Hib) conjugate vaccine was introduced into the UK's routine childhood immunisation schedule in 1992. As a result, the incidence of invasive disease declined to less than $5 \%$ of its previous level by $1998 .{ }^{1}$ However, episodes of Hib disease in fully vaccinated children do occur. ${ }^{2,3}$ The two commonest types of infection in such cases are meningitis, followed by epiglottitis, with other clinical presentations seen more rarely. ${ }^{3}$

$\mathrm{Hib}$ conjugate vaccine failure is likely to have multiple and complex causes. ${ }^{4}$ It may be linked to mounting a low antibody response to $\mathrm{Hib},{ }^{4}$ which has been documented in around one-third of cases studied. ${ }^{2}$ Associations with a number of predisposing conditions have also been described, particularly immunoglobulin deficiencies, as well as prematurity, Down's syndrome, and chromosomal abnormalities. ${ }^{3}$ Nonetheless, the majority of instances occur in children who appear otherwise entirely healthy. ${ }^{3}$

While unidentified, subtle immune deficiencies may be involved in some cases, ${ }^{3}$ Hib vaccine failure in apparently healthy children could also result from the effects of errors in production, storage, or administration on the vaccine's effectiveness. ${ }^{2}$ This report describes a case of Hib vaccine failure in which injection technique may have played an important role.

\section{CASE HISTORY}

A previously healthy 4-year-old girl was brought to accident and emergency with acute shortness of breath, 2 days after onset of coryzal symptoms. On examination she had obvious stridor, with difficulty swallowing oral secretions. She was tachycardic, and becoming increasingly drowsy. Poor response to treatment with nebulised salbutamol, budesonide, and adrenaline prompted referral to anaesthetics and ear, nose, and throat (ENT) for fibre-optic nasendoscopy, which demonstrated a red, swollen epiglottis. She was intubated then stabilised with adrenaline, steroids, and broad-spectrum antibiotic treatment, before transfer to the regional paediatric intensive care unit the next morning.

The local consultant in public health medicine (health protection) was notified of the case. The following day, microbiology reported heavy growth of 


\section{How this fits in}

This case, in which gluteal injection was associated with Hib vaccination failure, is consistent with existing evidence that gluteal administration of hepatitis $\mathrm{B}$ and rabies vaccines can result in low immunogenicity. For professionals involved in immunisation, it reaffirms the importance of ensuring that they and their colleagues remain adherent to current practice guidelines against the use of the buttocks as an injection site. Doing so should ensure that the risk of vaccination failure, and hence of children being left vulnerable to serious infection, is minimised.

Haemophilus influenzae type $\mathrm{b}$ on a throat swab culture. Discussion with the family and review of GP records confirmed that the patient had received routine $\mathrm{Hib}$ vaccinations at 2,3 , and 4 months of age, as well as a fourth dose at around 6 months as part of the 2003 booster catch-up campaign. Each of these was injected into the gluteal muscles.

The patient was commenced on ceftriaxone for treatment of Hib epiglottitis. She made an uneventful recovery, and was well enough to be discharged home 10 days later. Her initial Hib antibody titre was suboptimal at $0.23 \mathrm{mg} / \mathrm{l}$, just above the minimum protective level of $0.15 \mathrm{mg} / \mathrm{l}$. A marked increase in antibody response was demonstrated following a subsequent booster vaccination.

\section{DISCUSSION}

MEDLINE, EMBASE, CINAHL, and the Cochrane databases were searched for evidence about possible causes of Hib vaccine failure, in light of the patient's history. Key search terms included epiglottitis, Haemophilus influenzae type b and Hib, immunisation, and vaccine administration, site, and failure.

The patient had none of the recognised congenital or developmental factors associated with vaccine failure. ${ }^{3}$ Her low convalescent Hib antibody levels might suggest a previously occult general immune defect, but her strong response to a subsequent booster tends to contradict this hypothesis. She received her Hib booster aged 6 months, which is 6 months earlier than the current recommended timing. ${ }^{1}$ This could have resulted in a weakened antibody response when she became infected by Hib.

It is also possible that some or all of the doses of $\mathrm{Hib}$ vaccine that the patient received were defective. Although invasive Hib disease is quite rare, the lack of other notifications associated in time or place with this case is evidence against batch failure. An isolated production or storage chain fault cannot be completely ruled out, however.

The effectiveness of the conjugate Hib vaccine could have been influenced by the use of gluteal injections. Reports of lower antibody responses following gluteal administration of both hepatitis B and rabies vaccines support the view that, compared with the anterolateral thigh or deltoid muscles, immunogenicity is worse when vaccines are injected into the buttocks. ${ }^{5,6}$ This effect has not previously been reported specifically for Hib, but may be the result, for any vaccine, of erroneously injecting subcutaneously instead of into the gluteal muscle. ${ }^{5,6}$

Ten months before the patient received her first dose of Hib vaccine, the Royal College of Paediatrics and Child Health (RCPCH) recommended that only the anterolateral thigh or deltoid be used for all intramuscular injections. ${ }^{7}$ However, the key reference guide to vaccination practice at this time was the second (1996) edition of Immunisation Against Infectious Disease (the 'Green Book'). This did not explicitly proscribe the gluteal site, despite acknowledging that the efficacy of hepatitis $B$ vaccine is reduced by injecting into fatty tissue in the buttock. ${ }^{8}$ The third edition of the Green Book, now regularly revised via the internet, does conform to the RCPCH guidance, but was not published until 2006. ${ }^{1}$

This case highlights the importance for immunisation practitioners, and for all health professionals, of continuously remaining alert to all relevant sources for the latest knowledge and expert recommendations. Doing so will enable established local practices based on potentially outdated or ambiguous guidance to be identified and challenged.

\section{Consent}

The patient's father gave consent for publication of this case history.

\section{Discuss this article}

Contribute and read comments about this article on the Discussion Forum: http://www.rcgp.org.uk/bjgp-discuss

\section{REFERENCES}

1. Salisbury D, Ramsay M, Noakes K, (eds). Immunisation against infectious disease. 3rd edn. London: The Stationery Office, 2006.

2. Heath PT, Booy R, Griffiths H, et al. Clinical and immunological risk factors associated with Haemophilus influenzae type b conjugate vaccine failure in childhood. Clin Infect Dis 2000; 31(4): 973-980. http://www.journals.uchicago.edu/doi/abs/10.1086/318132 (accessed 18 Mar 2009).

3. Heath PT, McVernon J. The UK Hib vaccine experience. Arch Disease Child 2002; 86(6): 396-399.

4. Breukels MA, Spanjaard L, Sanders LAM, Rijkers GT. Immunological characterization of conjugated Haemophilus influenzae type b vaccine failure in infants. Clin Infect Dis 2001; 32(12): 1700-1705.

5. Shaw FE, Guess HA, Roets JM, et al. Effect of anatomic injection site, age and smoking on the immune response to hepatitis B vaccination. Vaccine 1989; 7(5): 425-430.

6. Centers for Disease Control and Prevention. Human rabies prevention - United States, 1999. Recommendations of the Advisory Committee on Immunization Practices (ACIP). MMWR Morb Mortal Wkly Rep 1999; 48(RR-1): 1-21.

7. Royal College of Paediatrics and Child Health. Position statement on injection technique. London: Royal College of Paediatrics and Child Health, 2002

8. Salisbury DM, Begg NT, (eds). Immunisation against infectious disease. 2nd edn. London: The Stationery Office, 1996. 